\title{
Corela
}

Cognition, représentation, langage

HS-6 | 2007

Cognition, discours, contextes

\section{Sources et tradition polonaises en linguistique cognitive}

\section{Arkadiusz Koselak}

\section{CpenEdition}

\section{Journals}

Édition électronique

URL : http://journals.openedition.org/corela/1494

DOI : $10.4000 /$ corela.1494

ISSN : $1638-573 \mathrm{X}$

\section{Éditeur}

Cercle linguistique du Centre et de I'Ouest - CerLICO

Référence électronique

Arkadiusz Koselak, « Sources et tradition polonaises en linguistique cognitive », Corela [En ligne], HS-6 | 2007, mis en ligne le 01 novembre 2007, consulté le 19 avril 2019. URL : http:// journals.openedition.org/corela/1494; DOI : 10.4000/corela.1494

Ce document a été généré automatiquement le 19 avril 2019

\section{(c) (i) (2)(2)}

Corela - cognition, représentation, langage est mis à disposition selon les termes de la licence Creative Commons Attribution - Pas d'Utilisation Commerciale - Partage dans les Mêmes Conditions 4.0 International. 


\title{
Sources et tradition polonaises en linguistique cognitive
}

\author{
Arkadiusz Koselak
}

Les grands postulats et méthodes de la linguistique cognitive se sont développés à partir des années soixante-dix, principalement sur la côte ouest des Etats-Unis mais la linguistique cognitive n'a pas été inventée à ce moment-là ; on peut penser au contraire qu'elle suit une tradition linguistique et philosophique déjà établie en Europe. E. Tabakowska (2004) fait le tour des linguistes polonais qui ont posé, et ce depuis le début du XXe siècle, des idées présentes dans la courant cognitiviste contemporain. Sa revue commence, pour la période pré-structurale, par Baudouin de Courtenay, J.M. Rozwadowski et B. Malinowski ; certains structuralistes polonais, comme L. Zawadowski, $\mathrm{J}$. Kuryłowicz et $\mathrm{D}$. Wesołowska étudiaient les faits linguistiques dans une perspective que l'on peut qualifier, du moins partiellement, de cognitive. Enfin, chez les linguistes contemporains, il faut citer T. Krzeszowski qui perfectionne l'Idealized Cognitive Model de G. Lakoff en y ajoutant l'indispensable composante axiologique et Anna Wierzbicka', laquelle est cognitiviste par ses postulats mais qui a développé une théorie originale et finalement assez radicalement différente $d u$ courant cognitiviste d'inspiration californienne.

2 Dans cet article j'aborde la tradition polonaise de trois points de vue : les origines, les divergences et les convergences. Tout d'abord, je m'intéresse à la sémantique de Jan $\mathrm{M}$. Rozwadowski qui, au début du XXe siècle, en plus d'avoir posé certaines principes généraux de l'approche cognitive, a développé des notions particulières que l'on retrouve ultérieurement chez R. Langacker, G. Lakoff et autres cognitivistes américains ; ensuite, je présente les principes de l'école ethnolinguistique polonaise qui, tout en s'inscrivant dans le courant cognitif par ses principes, en diverge par l'originalité de son objet ; enfin, dans une perspective de convergence, je m'intéresse à la problématique axiologique de $\mathrm{T}$. Krzeszowski qui, se situant dans le courant cognitif d'inspiration américaine, perfectionne l'Idealized Cognitif Model de G. Lakoff en y introduisant l'indispensable composante axiologique. 


\section{Un cognitiviste avant l'heure - Jan M. Rozwadowski}

3 Jan Michał Rozwadowski (1867-1935) était élève du psychologue allemand Wilhelm Wundt et une bonne partie de sa théorie linguistique prend sa source dans la théorie psychologique de son maître. Il a laissé derrière lui un nombre considérable d'écrits portant sur les problèmes divers concernant les sciences du langage et qui anticipent certaines développements de la linguistique et notamment l'avènement du courant cognitiviste.

Pour Rokoszowa (1991) la force et l'intérêt des idées de Rozwadowski réside dans le fait qu'il était à la marge de courants dominants de son époque. Rozwadowski était en dehors $\mathrm{du}$ structuralisme de son époque pour au moins deux raisons très importantes. Premièrement, il récusait l'étude du langage en tant qu'entité immanente et posait l'homme, en tant qu'individu, en tant qu'être cognitif ${ }^{2}$, percevant le monde et transformant le monde, au centre d'une théorie du langage. Cette prise de conscience de l'importance de l'homme et des processus cognitifs a amené Rozwadowski à s'interroger sur le statut des sciences du langage et de leur place parmi d'autres sciences. Compte tenu du fait que la langue est à la fois une réalité physique et une réalité culturelle, les sciences du langage sont à la fois des sciences exactes et des sciences humaines et la linguistique une science ayant un statut particulier, dépendante des autres mais aussi indépendante. Logiquement, Rozwadowski prône l'interdisciplinarité, si importante pour les cognitivistes d'aujourd'hui alors que le structuralisme de son époque se coupait du reste du monde scientifique en abstrayant la langue de l'homme. Deuxièmement, Rozwadowski refusait le figement structural de la langue en synchronie et considérait l'aspect évolutif du langage comme primordial pour comprendre sa dynamique, en même temps que cet aspect permet de considérer, grâce à l'évolution, le développement de la pensée de l'homme. Le langage est dynamique par excellence, il s'adapte à des changements de rapports entre l'homme et le monde.

En s'interrogeant sur la place des sciences du langage parmi les autres disciplines scientifiques, Rozwadowski réfléchit également à la cohérence interne de la linguistique elle-même. Au lieu de garder les domaines traditionnels, dont les appellations: phonétique, morphologie et syntaxe ne sont " pas claires et déformantes » (1950:199), il propose de parler tout simplement de phonèmes, de mots et d'énoncés ${ }^{3}$. Il met en garde contre les séparations de domaines et, tout comme Langacker et Wierzbicka des décennies plus tard, il considère qu'il faut étudier les faits linguistiques comme «une chaîne ininterrompue d'entités, des plus petites aux plus grandes, en ne perdant jamais de vue leur forme et leur sens » (ibid.). Ainsi tous les faits linguistiques ont un sens et si Rozwadowski parle le plus souvent des unités lexicales, il n'oublie pas pour autant la syntaxe qui, selon lui «encore plus directement, exige de faire le rapport entre ces faits et les évènements psychologiques » (1903: 35). Quant à la sémantique ${ }^{4}$, sa particularité découle davantage de la perspective et des rapports entre la forme et le sens différents que de la nature différente de l'objet étudié :

Cet état de choses indique clairement que la sémantique ne sera jamais une science du type de la phonétique ou de la morphologie qui ont avant tout affaire avec des faits linguistiques externes. Bien évidemment cela ne signifie pas que les faits linguistiques dont s'occupe la phonétique, et surtout la morphologie, n'ont pas de sens psychique. Mais le rapport du chercheur envers les faits phonétiques est tout à 
fait le contraire de celui des faits sémantiques: là il a affaire avant tout à des évènements physiologiques, ici à des évènements psychologiques. $(1903: 18)^{5}$

Tout comme les cognitivistes d'aujourd'hui, Rozwadowski établit un lien important entre la langue et la pensée. Il demeure néanmoins prudent et précise que la parole n'est pas la même chose que la pensée :

La parole ${ }^{6}$ 'est pas la même chose que la pensée, la seule pensée, et les mots ne sont pas uniquement les concepts, ne serait-ce parce que le début relatif du langage, encore visible aujourd'hui est la vie affective ; mais quoi qu'il en soit, le mot est à la pensée ce que l'ossature est au corps, et la langue en entier représente pour la pensée humaine comme un grand répertoire ou un dictionnaire bien rangé. (Rozwadowski, 1950 : 96)

7 Rozwadowski refusait les affirmations téléologiques énonçant que la langue exprimait la pensée et présupposant que la langue était en dehors de l'homme, distincte de la pensée et de la vie psychique en générale :

La parole "ne sert pas à exprimer ", mais tout simplement elle exprime, dans le sens où la face physique et la face psychique des processus font unité, ce qui relègue les notions de but et de moyen ${ }^{7}$ au rang secondaire. (ibid.)

8 L'importance de la non séparation de la langue et de la pensée transparait également dans le fait que la langue ne sert pas uniquement à communiquer :

[...] la langue n'exprime pas seulement, c'est-à-dire ne matérialise pas les pensée et les affects de l'homme pour les autres, mais aussi pour l'homme lui-même, ou autrement dit, les gens ne font pas que communiquer à l'aide de la langue, grâce à lui ils fixent également et notent des processus internes et des contenus, ce qui apporte une aide importante au développement intellectuel. (ibid.) ${ }^{8}$

Rozwadowski postule que c'est grâce à la langue que l'on peut le plus facilement étudier " la mécanique de la vie psychique » (op.cit. : 211). Tout comme les cognitivistes contemporains ${ }^{9}$, Rozwadowski souligne les bases expérientielles de la langue :

Dans la langue sont déposés, c'est-à-dire saisis, marqués et figés les éléments de toute la conscience et de toute la vie psychique humaine, de toute la vie "théorique » et "pratique »; en bref, les contenus élémentaires et les créations de toute « l'expérience » humaine vécue. (op.cit., 208)

10 La prise de conscience des rapports entre les processus cognitifs et la langue permet à Rozwadowski de pressentir la notion de construal mise en évidence ultérieurement par Langacker (1988), notion que Rozwadowski inclut dans le sens : «Il est donc évident que la langue, dans son sens objectif, est une réserve de mots, de formes et d'expressions; elle est également la création d'une compétence linguistique acquise et développée, située dans l'âme, renvoyant aux mécanismes psychologiques correspondants $»^{10}$ (Rozwadowski, op.cit. : 13) 11 .

11 En considérant l'unité linguistique comme un concept, Rozwadowski développe la notion de perception et arrive à la notion de saillance, si souvent utilisée aujourd'hui :

Le mot signifie donc toujours un concept complexe, dont une partie est perçue, au moment de le nommer, comme dominante: l'image phonétique se fige avec cet élément dominant dans une complication permanente, avec laquelle tous les autres caractéristiques permanentes et variables sont associées comme relativement moins saillantes. (1903: 46)

12 Partant de la psychologie de Wundt, Rozwadowski va encore plus loin dans ses réflexions sur les propriétés de la perception: il pose des mécanismes que Langacker (1988) appellera plus tard les relations profil/base et même les notions de gestalt et de point de vue: 
L'essence de cet évènement [nommer les choses] se base sur les propriétés de la perception: son étroitesse fait qu'on perçoit plus distinctement une partie seulement des éléments. Ainsi le nom d'une chose est le résultat d'une certaine partie bien délimitée de son concept que nous appellerons la caractéristique dominante, c'est-à-dire un concept partiel qui se trouve, au moment de la perception, dans le point de vue (Blickpunkt). L'unité de la perception fait que le contenu de la conscience perçu à un moment donné, qui se compose de différentes parties, est homogène, se présente comme un seul concept. (ibid.) ${ }^{12}$ perspective contrastive, en prenant pour exemple les expressions polonaise et française concernant l'acte de prendre quelque chose dans la poche qui se dit en polonais prendre quelque chose de la poche. Il conclut qu'en français on exprime (Rozwadowski, $1950: 47$ ) le moment initial de l'acte alors qu'en polonais on exprime le moment de sortie de la poche.

Un autre concept important de la linguistique cognitive contemporaine sur leque travaillait Rozwadowski est la métaphore. Bien que Rozwadowski ne soit pas le premier à postuler la métaphore en tant que mécanisme cognitif ${ }^{13}$, son approche de ce phénomène est très actuelle et mérite qu'on la rappelle ici. Pour Rozwadowski les métaphores sont un mécanisme omniprésent $(1903: 182)$ et indispensable, qui nous permet de penser et de parler :

On a dit déjà il y a longtemps que la langue est un ensemble de métaphores jaunies, mais ce n'est qu'une partie de la vérité car la plus grande partie de ce que nous appelons aujourd'hui métaphore, n'était pas métaphore. Encore aujourd'hui, dans la phase supérieure de son développement, l'homme ne peut penser ni parler autrement de beaucoup de choses : tout ce qui se meut, une montre, le soleil, l'eau $\mathrm{va}$, court, se déplace de la même manière que les gens et les animaux. $(1950: 39)^{14}$

Rozwadowski considère que l'on traite les mots abstraits de la même manière que les mots concrets, le traitement métaphorique des uns et des autres est donc identique. Une autre observation très importante de Rozwadowski sur les métaphores porte sur leur conventionnalisation :

Car toutes ces dites métaphores se dissolvent en brouillard lorsqu'on les observe de près, c'est-à-dire la frontière entre elles et les changements de sens que nous avons observés jusqu'ici s'efface complètement: [...] le rideau de la nuit est tout aussi indispensable que le cou de la bouteille. Sinon, comment l'homme pouvait et peut percevoir? (1903: 82).

Pour conclure avec cette présentation de la pensée originale de Rozwadowski, je citerai Rokoszowa (1991 : 23) :

Aujourd'hui, en lisant Rozwadowski, nous ne trouvons pas de fausses affirmations que nous serions enclins à rejeter. Nous prenons conscience au contraire que nous nous trouvons toujours dans les cercles dessinés par sa pensée par rapport à laquelle, la modernité n'a apporté vraiment que le perfectionnement de l'outil.

Rozwadowski a pressenti le cognitivisme et en a posé certains principes qui sont toujours actuels. Certaines de ses idées n'ont pas été développées suffisamment mais il a le mérite de les avoir formulées. Il écrivait principalement en polonais, il n'est pas accessible à la grande majorité de linguistes occidentaux et c'est certainement pour cette raison que l'on ne le retrouve pas dans les bibliographies des auteurs cognitivistes contemporains. L'évoquer aujourd'hui c'est aussi lui redonner la place qu'il devrait occuper dans l'histoire des sciences du langage. 


\section{Une perspective divergente - l'école ethnolinguistique de Lublin}

18 Tabakowska (2004: 24) en faisant sa revue des linguistes polonais concernés par la linguistique cognitive observe qu'ils « représentent un camp modéré qui essaie de plus en plus souvent d'introduire dans le nouveau paradigme ses propres problématiques de recherche ». L'école ethnolinguistique polonaise qui revendique son appartenance au courant cognitif (DĄbrowska \& Anusiewicz, 2000: 9; Tokarski, 1997/98) développe justement une approche originale en mettant l'accent d'une part sur les postulats cognitivistes, et d'autre part sur les postulats relativistes, principalement de la tradition allemande.

19 Partant de l'étude des parlers populaires et s'occupant actuellement tout aussi bien de la langue standard, l'objet de l'éthnolinguistique polonaise est l'image linguistique du monde [jĘzykowy obraz świata] dans la tradition néo-humboldtienne allemande ${ }^{15}$ (Anusiewicz, DĄbrowska et Fleischer, 2000 :11 ; Bartmiński, 2004). Bartmiński (2003 : 63), un des chefs de fil et fondateur de ce courant définit l'image linguistique du monde de la manière suivante :

L'ensemble d'idées fixées (stéréotypiques) sur l'homme et le monde pouvant être reconstruites à partir de données linguistiques de différents types: le système grammatical (les catégories flexionnelles, morphologiques, syntaxiques et textuelles), le système sémantique (le sens des mots et des unités complexes), les usages, et également les éléments "périlinguistiques", les croyances et les attitudes, toutes données qui peuvent se révéler pertinentes lors d'une communication effective.

20 Une précision terminologique s'impose ici et elle est d'autant plus importante qu'elle permet de dégager la spécificité de l'école polonaise dans le courant cognitif contemporain. Bartmiński (1999: 103) distingue deux variantes de l'étude de l'image linguistique du monde : en tant que vision du monde qui implique quelqu'un qui regarde, cette variante a surtout pour objet d'étude le sujet, tandis que l'image du monde, étant de l'ordre du résultat de la vision s'occupe surtout de l'objet. Autrement dit, comme le reformule Maćkiewicz (1999b: 23), les cognitivistes s'intéressent aux processus dynamiques de la vision alors que l'école polonaise s'intéresse davantage à l'objet statique, au produit de la vision. La différence va plus loin, dans la mesure où l'on pourra faire l'abstraction du sujet lorsqu'on aborde l'image :

Par l'image linguistique du monde il faudrait comprendre un modèle de la réalité idéalisé et abstrait, fonctionnant comme la composante de la compétence linguistique. En le comprenant ainsi, le terme linguistique a le sens de 'contenu dans la langue standard', et puisque la langue standard n'a pas d'existence réelle, mais qu'elle est un modèle abstrait construit sur la base des énoncés concrets, l'image linguistique du monde est aussi une abstraction qui existe à travers ses concrétisations idiolectales et textuelles.

21 Sans nier les variations linguistiques, n'oublions pas que l'école éthnolinguistique a étudié tout d'abord l'image linguistique du monde dans les parlers populaires, les chercheurs de cette école s'attachent principalement à étudier en quelque sorte l'invariant de l'invariant. Soulignons que l'image linguistique du monde concerne la connaissance naïve du monde ou la connaissance des locuteurs naïfs. Il ne s'agit pas d'un reflet du monde, mais d'une interprétation du monde et comme toute interprétation, 
l'image linguistique du monde est incomplète, elle correspond aux intérêts d'une communauté parlante.

De tradition néo-humboldtienne, l'éthnolinguistique polonaise est plus ancienne que le cognitivisme contemporain mais les chercheurs de cette école se sont tournés progressivement vers l'approche cognitive pour plusieurs raisons. Une des premières raisons est la prise en charge, par le cognitivisme, de l'homme dans sa totalité, donc également dans son fonctionnement culturel. Cette posture revient à rejeter l'immanentisme structuraliste. Deuxièmement, l'école éthnolinguistique, tout comme le cognitivisme, accepte les principes du caractère encyclopédique de la sémantique. La troisième raison tient à ce que les deux courants se fondent sur les principes de la catégorisation prototypique.

L'unité de base dans la problématique de l'école éthnolinguistique est le stéréotype. Dans la tradition de W. Lippman et H. Puttman (Bartmiński, 2004), il s'agit : « des liens fixés, c'està-dire reproduits et non crées selon le besoin, établis dans la mémoire collective au niveau concret qui correspond aux lexèmes » (Bartmiński, 1985 : 51). Le stéréotype est décrit à l'aide d'une définition cognitive. Le stéréotype est utilisé depuis longtemps dans les sciences sociales. Pourtant son usage en linguistique a rencontré un certain nombre de résistances. On peut s'en étonner dans la mesure où le stéréotype se définit comme une activité humaine et langagière de type régulatoire qui fonctionne de façon répétée et dont la structure est prévisible (Bartmiński, 1985). Bartmiński parle bien d'une activité dans la mesure où stéréotype est une répétition d'un certain mode de fonctionnement. Le résultat de cette activité devient une entité. L'entité cognitive qu'est le stéréotype n'est pas en ensemble lâche de connaissance mais il est organisé en aspects (Zinken, 2004: 116). La définition cognitive obtenue contient l'invariant central que l'on retrouve dans tous les emplois, ainsi que de traits actualisés dans les différents contextes, jusqu'aux connotations rares, activées dans la poésie. Ces dernières, bien que rares, participent pleinement à la cohérence interne du concept défini. Pour donner un exemple, la définition cognitive du chat en polonais établie par Anusiewicz (1994 : 139) se présente ainsi :

un animal domestique pas trop grand, avec des griffes aiguisées, incurvées et préhensibles, une bonne vue, une tête ronde bien formée, le corps agile et svelte couvert des poils doux et épais; agréable et joueur - pour cette raison élevé par l'homme pour le plaisir, et pour chasser les souris et les rats; ne supporte pas les chiens; ronronne et miaule, aime le lait; chasse en solitaire; silencieux et se déplace sans bruit. On lui attribue des caractéristiques comme : la ruse, la sagesse, la prudence, l'indépendance, l'autonomie ${ }^{16}$. A cause de sa grâce et de sa douceur, ainsi que les sensations agréables que provoque le contact avec sa fourrure douce et épaisse, comme à cause de son utilité, l'homme lui témoigne de la sympathie et d'une attitude positive.

Cette définition qui a été obtenue par l'analyse des occurrences linguistiques de chat, elle est cohérente et elle est, selon les critères de l'auteur, cognitivement plausible ${ }^{17}$.

Nous avons vu la différence majeure entre le cognitivisme et l'école éthnolinguistique polonaise qui est la différence de l'objet d'investigations. Pour redire les choses autrement, le cognitivisme privilégie, comme objet d'étude, le comment de la conceptualisation, alors que l'éthnolinguistique polonaise cherche à comprendre davantage le quoi. Tandis que la linguistique cognitive essaie d'établir les mécanismes et les schémas qui sous-tendent le fonctionnement linguistique et donc mental, l'école éthnolinguistique polonaise cherche à établir les définitions cognitives telles qu'elles sont construites dans l'esprit des locuteurs ${ }^{18}$. Nous avons vu également des convergences dans 
les postulats principaux. Nous allons maintenant aborder une différence de détail - un traitement différent de la notion du profil.

Sans entrer dans les détails de l'appareil méthodologique de Langacker (1988), on peut dire que profiler consiste à distinguer, dans une base conceptuelle, des structures sémantiques - des prédications. Pour dire les choses plus simplement, il s'agit de mettre certains éléments de base au premier plan et d'en laisser d'autres à l'arrière plan. On peut dire que chez Langacker le profil sert à construire des concepts, en comprenant le concept comme un certain profil d'une base. Il s'agit d'une opération mentale fondamentale. Dans cette conception de profil, il ne s'agit pas d'une relation entre une expression linguistique et un objet $\mathrm{du}$ monde, mais une relation entre la conceptualisation en entier et certains de ses fragments (Majer-Baranowska, 2004). Il s'agit d'une opération cognitive du conceptualisateur en tant qu'individu, dans son fonctionnement expérientiel. Autrement dit, la conceptualisation est indissociable de processus perceptuels de l'individu.

Bartmiński appréhende la notion de profil différemment. Pour lui il s'agit d'une relation entre la langue et le monde. Chez ce linguiste, l'action de profiler opère sur un concept déjà formé, il s'agit de distinguer dans un concept, certains de ses aspects - certains de ses profils. D'après Grzegorczykowa (1998), la conception de profil de Bartmiński permet de rendre compte des mécanismes de la création de nouvelles valeurs d'une unité et des variantes de sens. Bartmiński (1993) définit lui-même l'action de profiler ainsi : "une opération subjective linguistico-conceptuelle qui consiste en la création d'une image d' objet en le montrant sous certains de ses aspects, comme par exemple: l'origine, les caractéristiques, l'apparence, les fonctions.", ou encore: "il ne s'agit pas d'une variante de sens mais d'une variante de conceptualisation d'un objet, crée par un choix de facettes ${ }^{19}$, leur ordonnancement selon les règles d'implication, leur saturation par le sens selon les connaissances correspondantes du monde » (Bartmiński \& Niebrzegowska, 1998 : 217).

28 Il en découle qu'un même objet peut être profilé de plusieurs manières différentes, ainsi par exemple eau peut être conceptualisée soit d'un point de vue ontique, comme un élément naturel, soit d'un point de vue fonctionnel, comme une boisson, soit comme un élément magique ou médicinal, etc. A chaque fois, on active un ensemble différent de facettes et un contenu différent de ces facettes. On peut donc dire que le profil dans la conception de Bartmiński est en rapport avec la catégorisation, tandis que le profil le plus typique pourra lui être considéré comme un prototype (Bartmiński, Niebrzegowska, 1998).

Enfin, le profil de Bartmiński relève, davantage que le profil de Langacker, d'une conscience sociale et concerne le niveau discursif et textuel. Bartmiński insiste en effet sur le caractère social et culturel des profils, les profils sont effectués par l'individu faisant partie d'une communauté culturelle, la culture se manifestant dans les textes et les discours. Pour sa part, Langacker s'occupe davantage de l'individu lui-même dans la construction des profils.

30 L'école ethnolinguistique polonaise de Lublin peut être considérée comme complémentaire de l'orthodoxie cognitiviste. Sous-tendue par les mêmes postulats de base, elle s'intéresse davantage à un objet différent : l'image linguistique du monde plutôt qu'elle ne recherche les mécanismes de sa création. Inversement, les cognitivistes d'inspiration américaine étudient plutôt les mécanismes en jeu, comme en témoigne déjà leur conception différente de la notion de profil. L'école ethnolinguistique polonaise s'intéresse explicitement aux rapports de la langue avec la culture, la langue étant un de 
média possibles de la culture, mais la culture n'étant pas égale à la langue, l'image linguistique du monde fait partie de l'image culturelle du monde. L'ethnolinguistique polonaise aborde ainsi l'homme de manière plus large que le courant cognitiviste orthodoxe qui, souvent, se cantonne à un mentalisme. Langacker (1994:26), propose le schéma suivant pour illustrer les rapports de la langue et de la culture :

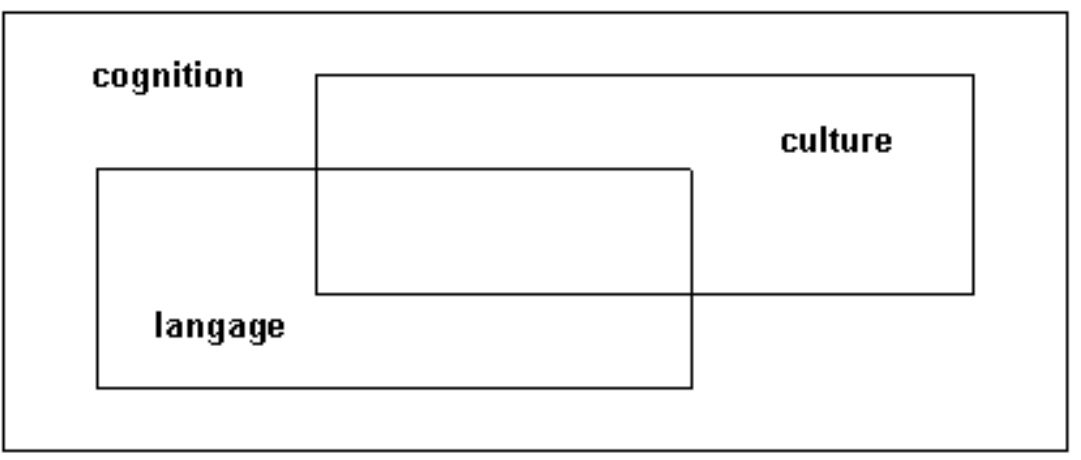

précisant qu'il existe des caractéristiques linguistiques comme par exemple la capacité d'articulation, qui ne font pas partie de la culture. L'école ethnolinguistique polonaise ne s'intéresse pas à des traits de ce type. Par ailleurs, elle n'envisage pas le langage en tant que tel mais l'image linguistique du monde. Selon cette vision les rapports langue culture peuvent être présentés de la manière suivante (en laissant de côté la cognition qui englobe le tout) :

l'image linguistique du monde

l'image culturelle du monde

On peut en extrapoler que la langue, en tant que système visant la significaiton, se situe à l'intérieur de la culture en général.

J. Anusiewicz (1994), linguiste polonais qui a participé à ce courant, a posé les principes d'une linguistique culturelle ${ }^{20}$ qui étudierait la problématique monde - homme - culture langue, nous pensons que le nom de linguistique intégrée serait adéquat.

\section{La sémantique, l'axiologie et l'Idealized Cognitive Model - T. Krzeszowski}

La dimension axiologique dans la sémantique se trouve au cœur des intérêts de $\mathrm{T}$. Krzeszowski qui lui a consacré un ouvrage entier en langue anglaise Angels and Devils in Hell. Se situant dans le courant cognitiviste, Krzeszowski $(1990,1994,1997)$ refuse la distinction entre le sens dénotatif et le sens connotatif, telle qu'elle a été conçue par les structuralistes et suit la proposition de G. Lakoff selon laquelle (1982) le sens des mots est organisé en Idealized Cognitive Models (ICM). Si l'on prend l'exemple des lexèmes chat et minou, on opposera la perspective structuraliste et cognitiviste ainsi : la première voir 
une différence de connotation (affective) entre les deux unités, la seconde en fait deux ICM distincts dont l'un diffère de l'autre par la charge axiologique (Krzeszowski, 1997).

Lakoff (1977) pose que les ICM ont les propriétés suivantes : ils constituent des ensembles structurés (gestalts), ils sont fondés sur les principes de catégorisation naturels, ils définissent des membres prototypiques des catégories. Pour Krzeszowski, les ICM doivent nécessairement contenir également les informations axiologiques, c'est-à-dire qu' on doit leur assigner une place dans l'échelle des valeurs, les informations axiologiques étant sémantiquement pertinentes.

Krzeszowski pose la nécessité de la prise en compte du paramètre axiologique en se fondant sur deux arguments majeurs. Premièrement, l'échelle axiologique bon - mauvais est une échelle de valeurs primaire et d'un très haut degré de généralitée ${ }^{21}$, elle subsume toutes les autres oppositions et notamment celle de la beauté et de la vérité (Krzeszowski $1990,1997)$; les enfants catégorisent tout d'abord les objets du monde en bons et mauvais du point de vue perceptif. Puis, l'échelle de la vérité ne peut être appliquée qu'au niveau des énoncés alors que l'échelle bon - mauvais peut être utilisée pour caractérisés des unités lexicales. Enfin, l'échelle bon - mauvais doit avoir un ancrage expérientiel dans le sens de Lakoff \& Johnson (1985), autrement dit le fait que bon se trouve plutôt vers le haut est determiné, d'une manière ou d'une autre, par l'expérience humaine dans la monde.

Se fondant sur la hiérarchie des valeurs de Tischner, Krzeszowski observe que l'opposition bon - mauvais n'est pas symétrique, mauvais semble exister comme un manque de bon. C'est en tout cas ce dont semble témoigner, dans beaucoup de langues, le caractère dérivé des lexèmes désignant les «mauvais » concepts, où les formes dérivées contiennent la négation de «bons » concepts, comme par exemple en français : déplaisir, insatisfait ${ }^{22}$. Selon Krzeszowski, l'existence de concepts négatifs indépendants comme enfer ou mauvais lui-même n'infirme pas la tendance générale.

Krzeszowski traite de manière originale la problématique de la spatialisation de l'échelle des valeurs. L'échelle de valeurs est horizontale, mais en rapport avec la théorie du fonctionnement métaphorique de Lakoff \& Johnson, Krzeszowski pose que bon s'associe de manière privilégiée à la direction vers le haut [up] et concerne ainsi l'échelle verticale. La dimension verticale s'associe au concept de la Grande Chaîne des Etres.

39 L'échelle bon - mauvais est une échelle, les concepts représentés par des unités lexicales peuvent se trouver à différents endroits du continuum, une partie de ce continuum est une zone floue, avec une charge axiologique relativement neutre, sans axiologie " ostensive », comme c'est le cas de verbes comme déclarer, comparer, écrire.

40 La dimension axiologique est préconceptuelle (Krzeszowski, 1994b) et elle «colore » en quelque sorte les autres schémas préconceptuels, ainsi par exemple l'existence a la valeur positive alors que l'expérience de base de la négation est le manque (l'absence); le caractère entier, le centre, le lien, l'intérieur, le but, le haut, la droite ont une valeur positive, alors que la partie, la périphérie, l'extérieur, le bas, la gauche ont la valeur négative.

41 L'évaluation axiologique en tant qu'un évènement cognitif s'apparente à la catégorisation, elle est donc aussi fondamentale que celle-ci et a le même statut ontologique et épistémologique. Les profils dans le domaine de valeurs s'effectuent conjointement aux profils dans d'autres domaines. Krzeszowski (1997) reprend les principes de la conceptualisation du sens de Langacker (1988) et les développe en y incluant la dimension axiologique : 
42 A : Le sens consiste en conceptualisation (expérience mentale), conceptualisation inclut l'évaluation axiologique.

B : Les structures sémantiques fréquemment utilisées en emplois typiques activent des réseaux de sens inter-reliés.

C. Les structures sémantiques se caractérisent par rapport aux domaines cognitifs, dont le domaine des valeurs, le domaine des valeurs est composé d'échelles axiologiques.

D. Une structure sémantique dérive sa valeur de la projection d'un profile sur une base, et sur le domaine axiologique. L'attribution d'une valeur à un concept ne peut pas être un mécanisme différent de l'attribution d'une valeur à la «chose" que ce concept représente.

E. Les structures sémantiques incorporent l'imagerie conventionnelle, c'est-à-dire : elles construisent une situation d'une manière particulière. Toutes les composantes de l'imagerie concernent également l'axiologie.

47 Le point le plus original proposé par Krzeszowski (1997) est la matrice axiologique fondamentale qui concerne notamment l'évaluation des groupes syntaxiques et donc des énoncés. Cette matrice se fonde sur les principes de la métaphorisation spatiale et utilise les concepts langackeriens de cible [trajector] et du site [landmark]. Elle peut être schématisée de la manière suivante :

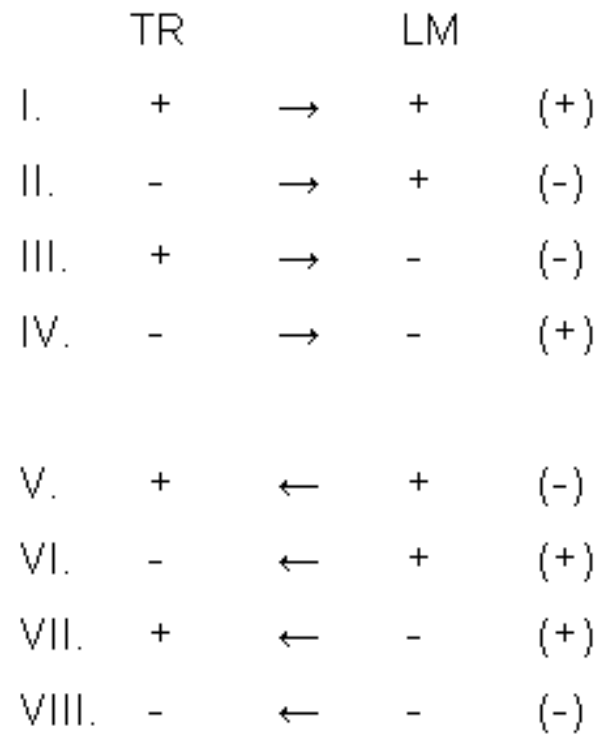

L'échelle axiologique fondamentale s'applique tout aussi bien aux relations statiques que dynamiques. Krzeszowski illustre la matrice axiologique fondamentale en partant du titre de son livre (Angels and Devils in Hell) et en s'appuyant sur la métaphore spatiale fondamentale, celle du conteneur, ainsi : 
Les constructions statiques:

$\begin{array}{lll}\text { I. } & \text { L'ange (+) au paradis (+) } & \text { positif } \\ \text { II. } & \text { Le diable (-) au paradis (+) } & \text { négatif } \\ \text { III. } & \text { L'ange (+) en enfer (-) } & \text { négatif } \\ \text { IV. } & \text { Le diable (-) en enfer (-) } & \text { positif } \\ \text { V. } & \text { L'ange (+) en dehors du paradis (+) } & \text { négatif } \\ \text { VI. } & \text { Le diable (-) en dehors du paradis (+) } & \text { positif } \\ \text { VII. } & \text { L'ange (+) en dehors de l'enfer (-) } & \text { positif } \\ \text { VIII. } & \text { Le diable (-) en dehors de l'enfer (-) } & \text { négatif } \\ \text { Les construction dynamiques: } & \\ \text { I. } & \text { L'ange (+) allant au paradis (+) } & \text { positif } \\ \text { II. } & \text { Le diable (-) allant au paradis (+) } & \text { négatif } \\ \text { III. } & \text { L'ange (+) allant en enfer (-) } & \text { négatif } \\ \text { IV. } & \text { Le diable (-) allant en enfer (-) } & \text { positif } \\ \text { V. } & \text { L'ange (+) quittant le paradis (+) } & \text { négatif } \\ \text { VI. } & \text { Le diable (-) quittant le paradis (+) } & \text { positif } \\ \text { VII. } & \text { L'ange (+) quittant l'enfer (-) } & \text { positif } \\ \text { VIII. } & \text { Le diable (-) quittant de l'enfer (-) } & \text { négatif }\end{array}$

Ces exemples, mêmes s'ils peuvent paraître un peu bizarres dans le discours quotidien, montrent qu'il n'y a pas besoin de faire la distinction entre les prédicats statiques et dynamiques du point de vue axiologique : les valeurs restent les mêmes dans les deux cas. Par ailleurs, la matrice axiologique fondamentale permet de prédire la charge axiologique des groupes syntaxique de tout types et des énoncés. Puzynina (2003) reproche à Krzeszowski de ne traiter que des relations spatiales, Krzeszowski (2003) considère que l'échelle axiologique fondamentale est applicable tout aussi bien à des relations non spatiales mais qu'il est important d'étudier le domaine spatial puisque beaucoup d'autres domaines se basent sur le domaine spatial concret par le biais de la métaphorisation.

Krzeszowski réintroduit l'axiologie dans le domaine de la linguistique cognitive en systématisant son approche. L'échelle axiologique fondamentale nécessite l'étude de son application dans d'autres domaines que le domaine spatial pour être validé, mais même si sa validité ne peut être maintenue que pour le domaine spatial, elle constitue une schématisation axiologique intéressante pour l'étude des valeurs en sémantique cognitive. 


\section{Conclusion}

51 Les anticipations de Rozwadowski, les développements originaux de l'école ethnolinguistique, les perfectionnements de Krzeszowski permettent de rendre compte d'une caractéristique épistémologique principale : l'histoire de science n'est pas linéaire, elle devrait plutôt être schématisée comme une spirale (cf. Tabakowska : 2004) avec des cercles qui se touchent parfois. D'où l'intérêt de retourner chez des auteurs comme Rozwadowski ou von Humboldt (Chabrolles-Cerretini, ce volume). Les développements originaux comme ceux de l'école éthnolinguistique polonaise et d'Anna Wierzbicka par exemple nous rappellent que le cognitivisme, bien que fondé sur un certain nombre des postulats communs ( $c f$. Geeraerts, 1991) est un ensemble hétéroclite d'approches du langage. C'est justement là que se trouvent sa richesse et sa puissance; et toute tentative de réduction à une orthodoxie, qu'elle soit californienne ou autre, serait préjudiciable pour le développement des sciences du langage, comme en 1920 le craignait déjà Rozwadowski.

\section{BIBLIOGRAPHIE}

ANUSIEWICZ J., 1994, Lingwistyka kulturowa, Wrocław : Wydawnictwa Uniwersytetu Wrocławskiego.

ANUSIEWiCZ J., DĄBRowsKa A., Fleischer M., 2000, JĘzykowy obraz świata i kultura. Projekt

koncepcji badawczej, JEzzyk a kultura, 13, pp.11-44.

ACHARD-BAYLE G., 2005, Polysémie de en : diversité et continuité des changements, in DENDALE P., Le mouvement dans la langue et la métalangue, Metz : Université de Metz, pp. 243-270.

BARTMIŃSKI J., 1985, Stereotyp jako przedmiot lingwistyki, Z problemów frazeologii polskiej i

słowiańskiej, III, pp. 25-53.

BARTMIŃSKI J., 1999, Punkt widzenia, perspektywa, jĘzykowy obraz świata, in BARTMIŃSKI J., (ed.), JĘzykowy obraz świata, Lublin : UMCS, pp. 103-120.

BARTMIŃSKI J., 2003, Miejsce wartości w jĘzykowym obrazie świata, in in BARTMIŃSKI J., JĘzyk w krĘgu wartości, Lublin : UMCS, pp. 59-86.

BARTMIŃSKI J., 2004, Etnolingwistyka słowiańska - próba bilansu, Etnolingwistyka, 16, pp. 9-27.

BARTMIŃSKI J., NIEBRZEGOWSKA, 1998, Profile a podmiotowa interpretacja świata, inBARTMIŃSKI J., TOKARSKI R., (eds) Profilowanie wjĘzyku i tekście, Lublin, UMCS, pp. 211-224.

CHABROLLE-CERRETINI A.-M., (ce volume),

DĄBRoWSKA A., ANUSIEWICZ J., 2000, WstĘp, JĘzyk a kultura, 13, pp. 9-10.

GEERAERTS D., 1991, La grammaire cognitive et l'histoire de la sémantique lexicale, Communications , 53, pp. 17-50. 
GRZEGORCZYKOWA R., 1998, Profilowanie a inne pojĘcia opisujĄce hierarchicznĄ strukturĘ znaczenia, in BARTMIŃSKI J., TOKARSKI R., Profilowanie wjęzyku i tekście, Lublin, UMCS, pp.9-18. JoHnson M., 1987, The body in the mind, Chicago/London, The University of Chicago Press. KRZESZOWSKI T., 1990, The axiological aspects of idealized cognitive models, in TOMASZCZYK J., LEWANDOWSKA-TOMASZCZYK B., Meaning and lexicography, Amesterdamp/Philadelphia : J. Benjamins, pp. 135-165.

KRZESZOWSKI T., 1994, Parametr aksjologiczny w przedpojęciowych schematach wyobrażeniowych, Etnolingwistyka, 6, pp. 29-51.

KRZESZOWSKI T., 1997, Angels and devils in hell, Warszawa : Energeia.

KRZESZOWSKI T., 2003, Jeszcze kilka słów o wartościach w filozofii i psychologii, in BARTMIŃSKI J., JĘzyk w krĘgu wartości, Lublin : UMCS, pp. 35-42.

LAKOFF G., JOHNSON M., 1985, Les métaphores dans la vie quotidienne, Paris : Minuit.

LANGACKER R., 1988, Foundations of cognitive grammar, Stanford: Stanford University Press.

LANGACKER R., 1994, Culture, cognition, and grammar, in Pütz M., Language contact and language conflict, Amsterdam/Philadelphie : J. Benjamins, pp.25-53

MAĆKIEWICZ , 1999, Co to jest językowy obraz świata?, Etnolingwistyka, 11, pp. 7-24.

MAJER-BARANOWSKA, 2004, Dwie koncepcje profilowania pojęć w lingwistyce, Etnolingwistyka, 16, pp. 85-109.

PUZYNINA J., 2003, Wokół jĘzyka wartości, in BARTMIŃSKI J., JĘzyk w krĘgu wartości, Lublin : UMCS, pp. 19-34.

RoKoszowa J., 1991, Problemy ogólnojęzykoznawcze i filozoficzne w pracach Jana Michała Rozwadowskiego, Biuletyn PTJ, XLIII-XLV, pp. 17-27.

ROZWADOWSKI M., 1903, Semazyologia czyli nauka o znaczeniu wyrazów. Jej stan obecny, zasady i zadania, Eos, pp.17-111

ROZWADOWSKI M., 1950, O zjawiskach i rozwoju jĘzyka, Kraków : KsiĘgarnia Gebethnera i Wolffa.

TABAKOWSKA E., 2004, Kognitywizm po polsku - wczoraj i dziś, Kraków : Uniwersitas.

TOKARSKI R., 1997/1998, JĘzykowy obraz świata a niektóre założenia kognitywizmu, Etnolingwistyka, 9/10, pp. 7-23.

WIERZBICKA A., 1985, Lexicography and conceptual analysis, Tucson: Karoma.

ZINKEN J., 2004, Metaphors, stereotypes, and the linguistic picture of the world: impulses from the Ethnolinguistic School of Lublin, metaphorik.de, 07, pp. 115-136, http://www.metaphorik.de/07/ zinken.pdf.

\section{ANNEXES}

\section{Anna Wierzbicka. Entre l'universel et culturel.}

Anna Wierzbicka a une position très originale dans la discussion abordée par AchardBayle (ce numéro) sur la tendance universaliste du courant cognitiviste d'inspiration américaine. Mon objectif n'est pas de présenter de manière exhaustive l'approche 
théorique lancée par Wierzbicka il y a une trentaine d'années, mais de situer son originalité par rapport à la linguistique cognitive californienne. Je ne vais pas discuter ici l'appartenance du courant wierzbickien à la linguistique cognitive, elle semble indiscutable ${ }^{23}$, notamment parce que le courant wierzbickien se fonde sur les postulats majeurs du cognitivisme comme le mentalisme, le primat de la sémantique et le principe d'organisation prototypique. Une grande différence sépare cependant le courant wierzbickien du cognitivisme classique : ce dernier s'occupe de mécanismes sous-tendant le fonctionnement du langage alors que le courant wierzbickien se donne comme l'objet d'étude les contenus conceptuels.

L'originalité de l'approche en MSN réside dans le fait qu'elle se construit entre deux courants apparemment contradictoires : l'universalisme et le relativisme. Mais ce paradoxe n'est qu'apparent, dans l'approche wierzbickienne l'universalisme et le relativisme ne se situant pas au même niveau.

L'approche en MSN, se fondant notamment sur Leibniz, postule l'existence d'un ensemble universel d'idées simples. Ces idées doivent avoir une réalisation linguistique, c'est-à-dire être exprimées par des unités linguistiques : qu'il s'agisse de lexèmes, de morphèmes liés ou de locutions, ce sont des primitifs sémantiques (semantic primes). On considère que ces idées sont « simples " parce que l'on ne peut les décomposer à l'aide d'unités plus simples que celles qui les expriment. Les primitifs sémantiques wierzbickiens transcendent l'opposition que F. Rastier (1991:142) fait entre les primitifs et les sèmes. Pour lui, le primitif est un concept qui appartient au langage ou à l'appareil cognitif, tandis que le sème est constitutif d'une langue. Les primitifs faisant partie de la MSN appartiennent autant au langage qu'à la langue, comme le revendiquent Goddard et Wierzbicka (2004: 154) : "Semantic primes are simple, indefinable meanings which appear to 'surface' as meanings of words or word-like expressions in all languages."

Les primitifs sont universels dans la mesure où on les suppose présents dans toutes les langues. Ce fait n'a bien évidemment pas été vérifié, les chercheurs en MSN étudient les langues appartenant aux différentes familles linguistiques pour étayer cette hypothèse. Notons que l'universalité d'un concept n'implique pas nécessairement son caractère primitif. Les primitifs composent la métalangue sémantique naturelle au sein de laquelle ils se combinent selon les principes d'une syntaxe naturelle. Cette syntaxe, qui n'a rien de commun avec les postulats chomskyens, consiste en : "universal pattern of combinability of the postulated primes, their semantic valency, so to speak" (Durst, $2003: 173$ ). Goddard (1997 : 201) exemplifie ce principe ainsi : si on prend un ensemble donné de primitifs, comme par exemple quelqu'un, quelquechose, dire, mauvais, et toi, la seule combinaison possible dans toutes les MSN sera : « quelqu'un a dit quelque chose de mauvais de toi ».

En résumé sur l'universalisme wierzbickien, on peut dire que tous les être humains possèdent un même ensemble d'idées simples. Ces idées sont matérialisées dans les langues à l'aide d'unités linguistiques qui se combinent selon les mêmes règles de syntaxe naturelle. Comme cela a été dit supra, il s'agit d'une problématique de contenus, mais on peut la mettre en parallèle avec les suppositions d'universalités de mécanismes cognitifs sous-tendant le fonctionnement des langues mis en évidence par les courants cognitivistes. Dans cette mesure, le cognitivisme classique et l'approche en MSN sont complémentaires.

Mais l'approche wierzbickienne va au-delà de l'universalisme en postulant en même temps un relativisme que C. Kerbrat-Orecchioni (2000 : 54) qualifie d'« extrême ». Une 
définition de sens donné par Wierzbicka (1994 : 226) permet de comprendre aisément comment ce relativisme est possible sur la base universaliste : "Meanings can be rigorously described and compared if they are recognized for what they are: unique and culture-specific configurations of universal semantic primitives." Ainsi les primitifs sont des outils, ou plutôt des « briques » pour reprendre la formulation leibnizienne utilisée par Wierzbicka, qui servent à construire les concepts plus complexes. Il est important de préciser que les explications verbales construites à l'aide des primitifs sémantiques doivent être de ce fait considérées comme des gestalts. L'extrémisme culturaliste dont parle Kerbrat-Orecchioni s'applique au fait que tous les faits de langue doivent être considérés a priori comme culturellement spécifiques, c'est-à-dire comme étant des configurations spécifiques des primitifs. Les études wierzbickiennes démontrent constamment la relativité culturelle de faits linguistiques, qu'ils soient lexicaux, grammaticaux ou pragmatiques. Le domaine lexical est le plus largement représenté, il s'en dégage l'étude de mots-clés qui sont les concepts particulièrement spécifiques d'une culture donnés (par exemple Wierzbicka 1997, 2006 ; Du Bartell, 2006), mais les domaines grammatical et pragmatique ne sont pas ignorés pour autant. Pour ne donner que quelques exemples, Wierzbicka (1988) démontre la relativité culturelle des structures syntaxiques concernant les actions corporelles, Enfield (2002) compile un ouvrage dans lequel les structures grammaticales de plusieurs langues sont étudiées du point de vue ethnosyntaxique ; enfin sont largement abordés les faits pragmatiques selon leurs spécificités culturelles (Wierzbicka, 2003 ; Goddard et Wierzbicka, 2004) ${ }^{24}$.

C'est justement à cause de cette possibilité d'être configurés de différentes manières, que les primitifs sémantiques, considérés comme la base universelle de la cognition humaine (et non seulement humaine par ailleurs, cf. Wierzbicka, 2003), que l'universalisme et le relativisme sont possibles au sein d'une même approche. L'existence de primitifs n'empêche pas la créativité, comme le croit Sériot (2005) après une lecture fragmentaire et trop rapide de Wierzbicka, mais bien au contraire, comme le considère A. Bogusławski (1991) : tout le monde peut apparemment penser et dire les choses comme il veut puisque chacun dispose d'un stock primitif de concepts et peut les combiner comme il veut moyennant un effort mental. Ainsi on peut dire que les spécificités culturelles (et individuelles également) sont construites sur la même base conceptuelle, qu'il y a une unité conceptuelle de l'espèce humaine. Cette unité est du même ordre que celle présupposée par les mécanismes « incarnés » par les cognitivistes d'inspiration californienne. Par contre, les primitifs sémantiques, en tant que le fondement de cette unicité, peuvent se combiner en un nombre très important de configurations. Les ensembles cohérents de ces configurations spécifiques forment des cultures. Unir dans une seule théorie l'universalisme et le relativisme est donc possible et ce n'est ni « malentendu » ni « une faille béante » (Sériot, 2005), mais la prise en compte des niveaux différents.

La linguistique cognitive californienne s'occupe de mécanismes cognitifs ; ces mécanismes étant dans une large mesure incarnés (embodied), on ne peut qu'être d'accord avec Rastier (cf. Achard-Bayle) lorsqu'il dit que la linguistique cognitive ignore le temps social. Elle étudie l'homme à travers la langue (et/ou le langage), mais l'homme en tant qu'être biologique. L'école wierzbickienne par contre étudie l'homme en tant qu'être social et donc culturel, elle cherche à comprendre l'homme à travers son contexte au sens large. Il s'ensuit des affinités différentes : tandis que les cognitivistes classiques se tournent vers la psychologie, les chercheurs de l'école wierzbickienne s'intéressent 
davantage aux travaux relevant de l'anthropologie. Les deux approches sont donc complémentaires, l'homme étant un être biologique et culturel. Le cognitivisme tend à supprimer le cloisonnement de domaines en sciences humaines, la linguistique cognitive elle-même couvre un champ d'investigations dans lequel il y a de la place pour tous les questionnements. Il faudra cependant envisager les outils d'une mise en commun pour que la linguistique cognitive ne soit pas un ensemble disparate d'analyses diverses.

DUBARTELL D., 2006, The development of a key word: The deictic field of Spanish crisis, in PEETERS B., (ed), Semantic Primes and Universal Grammar. Empirical evidence from the Romance languages, Amsterdam: J. Benjamins, pp. 259-288.

DURST U., 2003, The Natural Semantic Metalanguage approach to linguistic meaning, Theoretical Linguistics, 29, 2003, pp. 157-200.

ENFIELD N.J., (ed), 2002, Ethnosyntax. Explorations in Grammar \& Culture, Oxford: Oxford University Press.

FUCHS C., 2004, Pour introduire à la linguistique cognitive, in FUCHS C, (ed), La linguistique cognitive, Paris : Ophrys/Editions de la MSH, pp.1-24.

GODDARD C., 1997, The universal syntax of semantic primitives, Language Sciences, 19/3, pp. 197-207.

GODDARD C., WIERZBICKA A., 2004, Cultural scripts: What are they and what are they good for?, Intercultural Pragmatics, 1-2, 153-166.

Kerbrat-Oreccioni C., 2000,

RASTIER F., 1991, Sémantique et recherches cognitives, Paris : PUF.

SÉRIOT P, 2005, Oxymore ou malentendu? Le relativisme universaliste de la métalangue sémantique naturelle universelle d'Anna Wierzbicka, Cahiers Ferdinand de Saussure, 58, pp. 23-43.

WIERZBICKA A., 1988b, The semantics of grammar, Amsterdam/Philadelphia: John Benjamins. WIERZBICKA A., 2003, Cross-cultural pragmatics: The semantics of human interactions, Cambridge: Cambridge University Press.

WIERZBICKA A., 1994c, Semantic Primitives and Semantic Fields, in LEHRER A., KITTAY E., New Essays in Semantic and Lexical Organization, Hillsdale: Lawrence Erlbaum Associates, pp. 209-227.

WIERZBICKA A., 1997, Understanding Cultures through Their Key Words, Oxford : Oxford University Press.

WIERZBICKA A, 2003, Conceptual primes in human languages and their analogues in animal communication and cognition, Language Sciences, 26, pp. 413-441.

WIERZBICKA A., 2006, English. Meaning and Culture, Oxford: Oxford University Press.

\section{NOTES}

1. La théorie de cette chercheuse n'a pas été abordée ici pour deux raisons : d'une part elle semble être beaucoup plus connue que les autres approches retenues ici ; d'autre part, parce qu'il 
n'est pas certain que l'on puisse la considérer encore comme une linguiste polonaise. Cependant, les objectifs et la dynamique de ce numéro appellent à en parler; je le fais en annexe.

2. Rozwadowski utilise souvent le terme poznanie. Ce nom déverbal est dérivé du verbe poznać qui signifie à peu près "connaître" mais avec une valeur aspectuelle différente: "arriver à connaître "; il regroupe aussi bien les processus de la connaissance que la connaissance ellemême, on peut donc le traduire par cognition.

3. Il faut précisé que énoncé chez Rozwadowski n'équivaut pas à phrase, il ne considère pas comme les structuralistes que la phrase est l'unité supérieure de la langue; pour lui l'unité supérieure au-delà du mot est la période [powiedzenie] (1950 : 197).

4. Le terme de sémantique, ou plutôt sémasiologie [semazyologia], est utilisé souvent, et dans ce cas, il a le sens de sémantique lexicale.

5. Toutes les traductions dans cet article ont été effectuées par mes soins.

6. Je traduis mowa par parole suivant la tradition structuraliste polonaise, il me semble que dans le cas de Rozwadowski il faille considérer parole tout simplement comme le fait de parler. Les distinctions saussuriennes ne semblent pas être pertinentes ici.

7. Autrement dit, la langue n'est pas un moyen d'expression, la langue est expression.

8. Soulignements d'origine.

9. Cf. notamment Lakoff \& Johnson (1980), Johnson (1987).

10. Je souligne.

11. L'ouvrage auquel je renvoie est un recueil des écrits de Rozwadowski, je choisis de renvoyer à cet ouvrage plutôt que de référer à des publications particulières, difficilement accessibles.

12. Soulignements d'origine.

13. Cf. Achard-Bayle (2005).

14. Soulignements d'origine.

15. Davantage que la tradition américaine. Comme le résume Bartmiński (2004), les chercheurs américains s'occupent de la langue dans la culture, tandis que les chercheurs polonais, inversement, étudient la culture dans la langue.

16. Nous n'avons pas traduit deux caractéristiques de la liste faute de savoir comment les traduire autrement que par des périphrases.

17. On peut lui reprocher toutefois d'être incomplète. Anusiewicz (op.cit. 138) considère que des caractéristiques comme l'étrangeté, le caractère démoniaque et mystérieux relèvent uniquement des stylisations artistiques avec quoi on ne peut pas être d'accord. Les liens entre les chats noirs et les malheurs sont évidents dans le polonais courant.

18. Ce grand principe rapproche l'école ethnolinguistique polonaise et Wierzbicka, des différences existant toutefois.

19. Les auteurs utilisent ici la notion de facette dans la conception de Wierzbicka (1985) : les facettes sont des ensembles structurés des aspects (caractéristiques) d'un concept. Bartmiński (1998) rapproche la notion de facette et la notion de domaine langackerien.

20. En polonais : lingwistyka kulturowa. Il n'y a pas, il nous semble, d'équivalent exact en français : il ne s'agit ni d'une linguistique culturelle ni d'une linguistique de la culture, mais d'une linguistique qui étudie la culture à travers la langue.

21. Les deux entrent dans la métalangue sémantique naturelle de Wierzbicka.

22. Dans les exemples de l'anglais Krzeszowski donne également happiness - unhappiness. On note, en français, que les lexèmes bonheur et malheur sont construits sur les primitifs bon - mauvais (mal).

23. Bien que certains semblent penser le contraire, elle est par exemple absente de publications généralistes de Fuchs (2004) au sujet de la linguistique cognitive.

24. On trouvera la bibliographie complète des études wierzbickiennes à http://www.une.edu.au/ $\mathrm{lcl} / \mathrm{nsm} /$ 


\section{RÉSUMÉS}

Les grands postulats et méthodes de la linguistique cognitive se sont développés à partir des années soixante-dix, principalement sur la côte ouest des Etats-Unis. Mais, contrairement à ce que certains aimeraient penser, la linguistique cognitive n'a pas été inventée à ce moment là, elle suit une tradition linguistique et philosophique européenne. L'article se propose de rendre compte de certains travaux polonais en lien avec la linguistique cognitive. E. Tabakowska (2004) présente ceux des linguistes polonais qui ont posé dès le début du XXe siècle certaines des idées que l'on retrouve aujourd'hui dans le courant cognitiviste contemporain. Sa revue commence, à la période pré-structurale, par Baudouin de Courtenay, J.M. Rozwadowski et B. Malinowski ; quant aux structuralistes polonais, certains, comme L. Zawadowski, J. Kuryłowicz i D. Wesołowska étudiaient les faits linguistiques d'une perspective que l'on peut qualifier, du moins partiellement, de cognitive. Enfin, chez les linguistes contemporains, il faut citer, entre autres, $\mathrm{T}$. Krzeszowski, E. DĄbrowska et E. Tabakowska elle-même. L'article aborde cette tradition polonaise du cognitivisme sous trois aspects successifs: les sources, les divergences mais aussi les convergences avec le courant américain. Tout d'abord, l'article renvoie à la sémantique de Jan Rozwadowski qui, au début du XXe siècle, a développé des principes théoriques et des notions particulières que l'on retrouve ultérieurement chez R. Langacker, G. Lakoff et d'autres ; ensuite, sont envisagés les postulats de l'école ethnolinguistique polonaise qui, tout en s'inscrivant dans le courant cognitif par ses principes, en diverge par l'originalité de son objet; enfin, dans une perspective de convergence, l'article présente la problématique axiologique de T. Krzeszowski qui, se situant dans le courant cognitif d'inspiration américaine, perfectionne l'Idealized Cognitive Model de G. Lakoff en y introduisant la composante axiologique.

The aim of the article is to give an account of certain Polish works relating to cognitive linguistics. The Polish cognitivist tradition is approached via three successive aspects: the sources, the divergences but also the convergences with the American school. To start with, the article refers to Jan Rozwadowski's semantics which, in the early $20^{\text {th }}$ century, developed the theoretical principles and particular notions later found in the works of R. Langacker, G. Lakoff and others. Secondly, the postulates of the Polish ethno-linguistic school are envisaged. This, while adhering to the cognitive current by its principles, diverges from it in the originality of its subject. Finally, in a convergent perspective, the article presents T. Krzeszowski's axiological problematic which, as part of the American-inspired cognitive current, perfects G. Lakoff's Idealized Cognitive Model by introducing the axiological component into it.

\section{INDEX}

Mots-clés : linguistique polonaise, image linguistique du monde, profils, axiologie

Keywords : polish linguistics, linguistic picture of the world, profiles, axiology 
AUTEUR

ARKADIUSZ KOSELAK

Université Paul Verlaine de Metz, CELTED 